\title{
Poly(ethylene oxide) Bionanocomposites Reinforced with Chitin Nanofiber Networks
}

\author{
Jie $\mathrm{Wu},{ }^{\dagger, \S}$ Haisheng Lin ${ }^{\ddagger} \S$ and J. Carson Meredith ${ }^{\ddagger *}$ \\ †School of Materials Science and Engineering, Georgia Institute of Technology, Atlanta, Georgia \\ 30332, United States, $\neq$ School of Chemical and Biomolecular Engineering, Georgia Institute of Tech- \\ nology, Atlanta, Georgia 30332, United States.
}

Keywords: Chitin, Nanofibers, Polymer nanocomposites, Poly(ethylene oxide)

\begin{abstract}
With excellent reinforcing performance and many remarkable inherent properties, such as renewability and high strength-per-unit-mass, chitin nanofibers (ChNFs) are attractive resources for polymer composite applications. However, significant challenges resulting from chitin's insolubility have hindered efforts to incorporate it effectively into polymer matrices. Here, ChNFs extracted from crab shells were used as fillers to reinforce polyethylene oxide (PEO). The dispersion of ChNFs in the polymer matrix and the interactions between fiber and matrix were studied by utilizing solvent-etching of the polymer matrix, FTIR spectroscopy and AFM colloidal probe adhesion measurements. The results show that the ChNFs were dispersed well and formed a fine interconnected network structure in the PEO matrix. ChNFs also exhibited strong adhesion with PEO, resulting from hydrogen bond and van der Waals forces. The ChNF interconnected network greatly enhanced the mechanical properties of PEO, with a 3-fold increase in both the tensile strength and elastic modulus of the nanocomposites at $20 \% \mathrm{ChNF}$ loading. Processing ChNFs in suspensions with slight acidity and water-soluble polymers appears to support the formation of highly interconnected networks that makes ChNF-polymer composites excellent candidates for reinforced, light-weight, renewable materials.
\end{abstract}

\section{Introduction}

The development of nanofiller-reinforced polymer composites has attracted intense attention from researchers over the past two decades due to the large surface area/volume ratio of nanofillers, and various materials have been utilized to enhance the mechanical properties of polymer matrices, such as singlewalled/multi-walled carbon nanotubes, layered silicate and nanocellulose.[1-7] To date, tremendous efforts have been devoted to improving filler adhesion with polymers and their dispersion in matrices since these two factors significantly influence stress transfer in nanocomposites. Methods applied to address these issues include physical-mechanical treatment and chemical functionalization.[2-7] However, it is still challenging to enhance particle or fiber dispersion and adhesion with the matrix simultaneously.[27]

${ }^{*}$ Corresponding Author:

E-mail address: carson.meredith@chbe.gatech.edu 
limit applications. These mechanical limitations and its water solubility make PEO an ideal model system for examining dispersion, adhesion and strengthening possible with ChNFs. In this work, we prepared ChNF-PEO composites with up to $20 \%$ ChNF content. Solvent-etching of the polymer matrix followed by SEM, FTIR spectroscopy and AFM colloidal probe adhesion measurements were used to study the ChNF dispersion and adhesion between ChNF and PEO matrix. The results demonstrate that without utilizing chemical surface modification, ChNFs can be readily dispersed in $\mathrm{PEO}$, form a remarkable interconnected network nanostructure and have strong adhesion with PEO, significantly enhancing tensile strength and elastic modulus simultaneously.

\section{Experimental Section}

\subsection{Materials}

Dried crab shell flakes were purchased from TCI America. Deionized water $(18.2 \mathrm{M} \Omega \mathrm{cm})$ was prepared in a Barnstead Easypure RoDi purification system. Hydrochloric acid, sodium hydroxide, acetone and ethanol were purchased from EMD Chemical Inc. Poly(ethylene oxide) (PEO, $M v=1,000,000$ $\mathrm{g} / \mathrm{mol}$, Sigma-Aldrich), poly(ethylene) (PE, $M w=$ 40,000 g/mol, Sigma-Aldrich), poly(styrene) (PS, $M w=230,000 \mathrm{~g} / \mathrm{mol}$, Sigma-Aldrich), poly(vinyl acetate) (PVAc, $M w=50,000 \mathrm{~g} / \mathrm{mol}$, Alfa Aesar), and poly(vinyl alcohol) (PVOH, $M w=89,000-98,000$ $\mathrm{g} / \mathrm{mol}$, Sigma-Aldrich) were used as received without further purification. 1, 2, 3-trichlorobenzene (TCB, Sigma-Aldrich), hexafluoroisopropanol (HFIP, TCI America), and toluene (Sigma-Aldrich), glycerol (Alfa Aesar, purity $>99 \%$ ) and diiodomethane (Alfa Aesar, purity $>99 \%$ ) were used as received. Polystyrene (PS) particle with diameters of $\sim 10 \mu \mathrm{m}$ was purchased from Alfa Aesar Inc.

\subsection{Methods}

\subsubsection{Preparation of ChNFs}

Dried crab shell flakes were processed to obtain purified chitin.[11, 12, 15, 16] Ground crab shells were refluxed in $5 \mathrm{wt} \%$ sodium hydroxide in DI water for $6 \mathrm{~h}$ to remove protein. The suspension was filtered and rinsed with DI water until the $\mathrm{pH}$ was 7 . Next, the filtered solids were treated with $7 \%$ hydrochloric acid for $6 \mathrm{~h}$ at room temperature to remove minerals. After filtration and washing with DI water, the treated sample was refluxed in a $5 \% \mathrm{NaOH}$ solution for 2 days to remove residual proteins. A fi- nal extraction with acetone and ethanol was used to remove any remaining residues (soluble dye for example). The purified chitin was dispersed in distilled water under acidic condition and then this mixture was passed through a high-pressure homogenizer (Bee International Inc., MA) to generate ChNFs (aqueous medium $\mathrm{pH}$ is $\sim 4.1$ ). Detailed information on ChNF production is described elsewhere.[11]

\subsubsection{Preparation of ChNF/PEO Nanocomposite Films}

The PEO was firstly dissolved at 2 wt.\% in DI water at room temperature under a magnetic stirring. To prepare 5, 10, 15 and $20 \mathrm{wt} . \% \mathrm{ChNF} / \mathrm{PEO}$ composite films, the proper amount of $\mathrm{ChNF} /$ water dispersions were added to the PEO solution. These mixtures were magnetically stirred for two days, and were subsequently casted into a PS Petri dish, followed by drying under vacuum at $40{ }^{\circ} \mathrm{C}$ for two days. The free standing dried $\mathrm{ChNF} / \mathrm{PEO}$ nanocomposite films were obtained by carefully peeling films from the PS substrate. Neat PEO films were prepared using the same processing conditions for comparison. Both neat PEO and composite films had a thickness of $\sim 50 \mu \mathrm{m}$, as determined by an interferometer (Model ID-C112CEB, Mitutoyo Corp.).

\subsubsection{Preparation of ChNF Coated Polystyrene (PS) Colloidal Particles}

In order to fabricate colloidal probes coated with ChNFs for adhesion measurements, a $0.5 \mathrm{wt}$ \% ChNF in water dispersion was added to a PS particle suspension (10 $\mu \mathrm{m}, 2.5 \mathrm{wt} \%$ in water), followed by agitation using a rotational shaker for $12 \mathrm{~h}$. The mixture was then centrifuged in a micro-centrifuge (VWR Micro 1207). The ChNF coated PS particles were settled at the bottom of the container and dried in air at room temperature.

\subsubsection{Preparation of Polymer Films}

Substrates for colloidal-probe adhesion studies were prepared by coating a series of polymer films on Piranha-cleaned silicon wafers. The PE solution was prepared by dissolving 5\% PE by mass in hot 1 , 2, 3-trichlorobenzene at $\sim 100^{\circ} \mathrm{C}$. The PS solution was prepared by dissolving $10 \%$ PS by mass in toluene. 5 wt. \% PVOH and PVAc solutions were prepared in hexafluoroisopropanol. The PEO solution was prepared by dissolving $1 \mathrm{wt}$. \% by mass in DI water. Polymer films were prepared on Piranhaetched silicon substrates, by using a knife-edge coat- 
ing method described in detail elsewhere.[17] The cast polymer films were firstly dried at room temperature for $24 \mathrm{~h}$ and then dried under vacuum for at least $12 \mathrm{~h}$ (PVAc at $20{ }^{\circ} \mathrm{C}$ for $48 \mathrm{~h}$ and other polymers at $60{ }^{\circ} \mathrm{C}$ for $12 \mathrm{~h}$ ) to remove the residual solvent. After drying, films were transferred to a desiccator and stored prior to measurements. Film thickness was approximately $10-20 \mu \mathrm{m}$, determined by using an interferometer (Model ID-C112CEB, Mitutoyo Corp.).

\subsubsection{Measurements of Adhesion Forces}

Adhesion force measurements were carried out using atomic force microscopy (AFM) (Veeco Dimension 3100). Tipless rectangular cantilevers with nominal spring constants of 0.6-3.7 N/m (Applied NanoStructures, Inc., Santa Clara, CA) were used. Single ChNF-coated or bare PS particles were glued to the tipless cantilevers with a small amount of epoxy resin (Epoxy Marine, Loctite, Westlake, $\mathrm{OH}$ USA) using a procedure described in detail elsewhere.[18] The actual spring constants for the cantilevers with the attached ChNF-coated or bare PS particles $(0.7-1.1 \mathrm{~N} / \mathrm{m})$ were determined directly by the methods of Burnham and Hutter et al..[19] A series of 20 force-distance curves were measured for each combination of PS/ChNF or bare PS tip-polymer surface, taken on three separate substrate surfaces within three randomly chosen $1 \mathrm{~cm} \times 1 \mathrm{~cm}$ areas on each substrate at $20{ }^{\circ} \mathrm{C}$, humidity $25-30 \%$. Three separate ChNF-coated or bare PS particle tips were used for each set of measurements with a given substrate. The applied load during force measurements was $2.5 \mathrm{nN}$. The mean $\left(R_{a}\right)$ and root-mean-square $(r m s)$ surface roughness of each polymer film for adhesion measurements were obtained from topography scans of three randomly-chosen $10 \mu \mathrm{m} \times 10 \mu \mathrm{m}$ areas on each polymer surface by using AFM with a standard pyramidal silicon nitride probe.

\subsubsection{Contact Angle Measurements}

Contact angles of each polymer surface were measured at $20{ }^{\circ} \mathrm{C}$ using a video contact angle system (AST products 2500XE, Billerica, MA). Three standard testing liquids were chosen, two polar liquids (DI-water and glycerol) and one nonpolar (diiodomethane), to calculate the surface tension components of each polymer surfaces. The water contact angles of those polymer surfaces were measured only 3-5 seconds after drop placement. Generally, we didn't observe a significant change of the contact angle un- til after at least 15 seconds, which presumably occurs due to dissolution of the substrate. Nine $1 \mu \mathrm{L}$ drops of each liquid were used for the contact angle test of each polymer surface. Surface tension components corresponding to van der Waals (VDW), Lewis acid, and Lewis basic interactions were calculated from measured contact angle data by using van Oss and Good's van der Waals acid-base theory.[20] According to this theory, the surface energy is accessed from Equation 1.

$$
\gamma_{S}=\gamma_{S}^{\mathrm{vW}}+2\left(\gamma_{S}^{+} \gamma_{S}\right)^{1 / 2}
$$

where, $\gamma_{S}$ is the total surface tension, $\gamma_{S}{ }^{\mathrm{vW}}$ is the van der Waals component, $\gamma_{S}{ }^{+}$is the acid (electron acceptor) component, and $\gamma_{S}{ }^{-}$is the base (electron donor) component of the solid substrate. The relation between surface energy components and the liquidsolid-air contact angle $(\theta)$ is given as:

$\gamma_{L}(1+\cos \theta)=2\left(\gamma_{S}^{\mathrm{vW}} \gamma_{L}^{\mathrm{vW}}\right)^{1 / 2}+2\left(\gamma_{S}^{+} \gamma_{L}^{-}\right)^{1 / 2}+2\left(\gamma_{L}^{+} \gamma_{S}^{-}\right)^{1 / 2}$

where, $\gamma_{L}$ represents the surface energy of the testing liquids. By using the known components of $\gamma_{L}$ for three carefully-chosen liquids, the $\gamma_{S}$ components can be determined by regression. The surface tensions of the testing liquids are as follows: water, $\gamma^{+}=\gamma^{-}=$ $25.5, \gamma^{\mathrm{vW}}=21.8, \gamma=72.8 \mathrm{~mJ} / \mathrm{m}^{2}$; glycerol, $\gamma^{+}=3.92$, $\gamma^{-}=57.4, \gamma^{\mathrm{vW}}=34.0, \gamma=64.0 \mathrm{~mJ} / \mathrm{m}^{2}$; diiodomethane, $\gamma^{+}=\gamma^{-}=0, \gamma^{\mathrm{vW}}=50.8, \gamma=50.8 \mathrm{~mJ} / \mathrm{m}^{2} .[21,22]$

\subsubsection{Characterizations of ChNFs}

The morphologies of the prepared materials were characterized using Field-Emission Scanning Electron Microscopy (SEM, Zeiss Ultra 60, Carl Zeiss SMT, Ltd., Thornwood, NY). Before imaging, these samples were coated with a thin layer of gold/palladium (Hummer IV Sputtering System) to prevent sample charging. A solvent-etching procedure was used to selectively remove PEO domains from composites in order to aid in visualization of the ChNF morphology. Etched films were prepared by washing with water followed by rinsing with ethanol and drying at room temperature for 2 days prior to SEM imaging.

The attenuated total reflectance-Fourier transform infrared spectra (ATR-FTIR) of ChNF, neat PEO and nanocomposite films were recorded using a Bruker Vertex 80v FTIR spectrometer coupled to a Hyperion 2000 IR microscope under a 20x magnification ATR objective (Bruker Optics, Inc., Billerica, MA). Measurements were collected from 4000 to $400 \mathrm{~cm}^{-1}$ with a resolution of $4 \mathrm{~cm}^{-1}$, and were averaged over 64 
scans. The surface charge of ChNFs at $\mathrm{pH} 4.1$ in water was measured by a Malvern Zetasizer Nano ZS 90 .

Mechanical characterization of neat PEO and PEO nanocomposites was conducted using a highthroughput mechanical characterization (HTMECH) apparatus under ambient conditions, described in detail elsewhere.[23, 24] Briefly, the polymer films were mounted in between two stainless steel plates and indented by a steel pin with a diameter of 1.5 $\mathrm{mm}$ at a constant strain rate $(0.5 \mathrm{~mm} / \mathrm{s})$, resulting in equi-biaxial deformation. For each sample, a minimum of 9 stress-strain tests were performed to obtain films' mechanical properties, such as elastic modulus and tensile strength.

The glass transition temperatures of the materials were measured using dynamic mechanical analysis (DMA, Q800, TA Instruments, DE USA). The samples were cut into rectangular strips about $\sim 3 \mathrm{~cm}$ long and $\sim 3 \mathrm{~mm}$ wide and were tested in a tension mode while being heated from $-90{ }^{\circ} \mathrm{C}$ to $40{ }^{\circ} \mathrm{C}$ with a heating rate of $2^{\circ} \mathrm{C} / \mathrm{min}$ at a frequency of $1 \mathrm{~Hz}$. All tests were performed in the linear viscoelastic region.

Differential scanning calorimetry (DSC) (Q200, TA Instruments, USA) was used to obtain the melting temperature and crystallinity of neat PEO and PEO nanocomposites. Approximately 5-10 $\mathrm{mg}$ of sample was loaded into aluminum pan. The PEO samples were heated and cooled at a rate of $10{ }^{\circ} \mathrm{C} / \mathrm{min}$ under a nitrogen flow of $50 \mathrm{~mL} / \mathrm{min}$. The samples were firstly cooled from room temperature to $-80{ }^{\circ} \mathrm{C}$, held at $-80{ }^{\circ} \mathrm{C}$ for $5 \mathrm{~min}$, and were heated to $120{ }^{\circ} \mathrm{C}$, followed by maintaining at this temperature for $5 \mathrm{~min}$ and cooling to room temperature.

\section{Results and Discussion}

\subsection{Morphologies of ChNF/PEO Nanocomposite Films}

ChNFs produced from the high-pressure homogenization process have an average diameter of $20 \mathrm{~nm}$, mainly ranging from 5 to $50 \mathrm{~nm}$ and lengths that vary between hundreds of nanometers to several micrometers, as shown in Figure S1. They have a zeta potential of $+57.5 \mathrm{mV}$ at $\mathrm{pH} 4.1$ due to protonated $-\mathrm{NH}_{3}{ }^{+}$ groups.[11] SEM images of the as-prepared films, shown as cross-section (Figure $1 \mathrm{~A}, \mathrm{~B}$, and Figure S2) and top-view (Figure 1 C, D) indicated that $10 \%$ and $20 \% \mathrm{ChNF}$ composites have void-free surfaces. The outlines of fibrous morphologies assumed to be ChNFs can be observable, suggesting that ChNFs were imbedded in the PEO matrix and had good adhesion with PEO. However, the identity, size and interconnectivity of the ChNFs cannot be observed directly from Figures 1 A-D. Since PEO is water soluble, but ChNFs are not, a solvent-etching procedure was used to selectively remove PEO domains in composites. As shown in supplementary data (Figure S3), neat PEO films were totally dissolved within 3 hours of water immersion, while $\mathrm{ChNF} / \mathrm{PEO}$ composites maintained their shape after 10 days of water immersion and 3 days of ethanol immersion before drying. Figure $1 \mathrm{E}$ and F show SEM images of the top surfaces of the dried solvent-etched $10 \%$ and $20 \%$ ChNF composite films, respectively. Porous fibrous network structures are observed in both samples, and most fibers in the $10 \% \mathrm{ChNF}$ sample (Figure $1 \mathrm{E}$ ) have diameters ranging from 5 to $50 \mathrm{~nm}$. Despite more fiber aggregates being observed for $20 \%$ $\mathrm{ChNF} / \mathrm{PEO}$, there are still many fibers with diameters of below $50 \mathrm{~nm}$ (Figure $1 \mathrm{~F}$ ). Figure 1 and S3 clearly show that the ChNFs were dispersed well and formed network structures in the PEO matrix. We propose that the good dispersion of ChNFs is ascribed to the excellent dispersion of ChNFs in water before their mixing with $\mathrm{PEO}$, electrostatic repulsion between ChNFs in PEO solution $(\mathrm{pH}<6)$, and strong adhesion between ChNF and PEO, as discussed below in detail. 

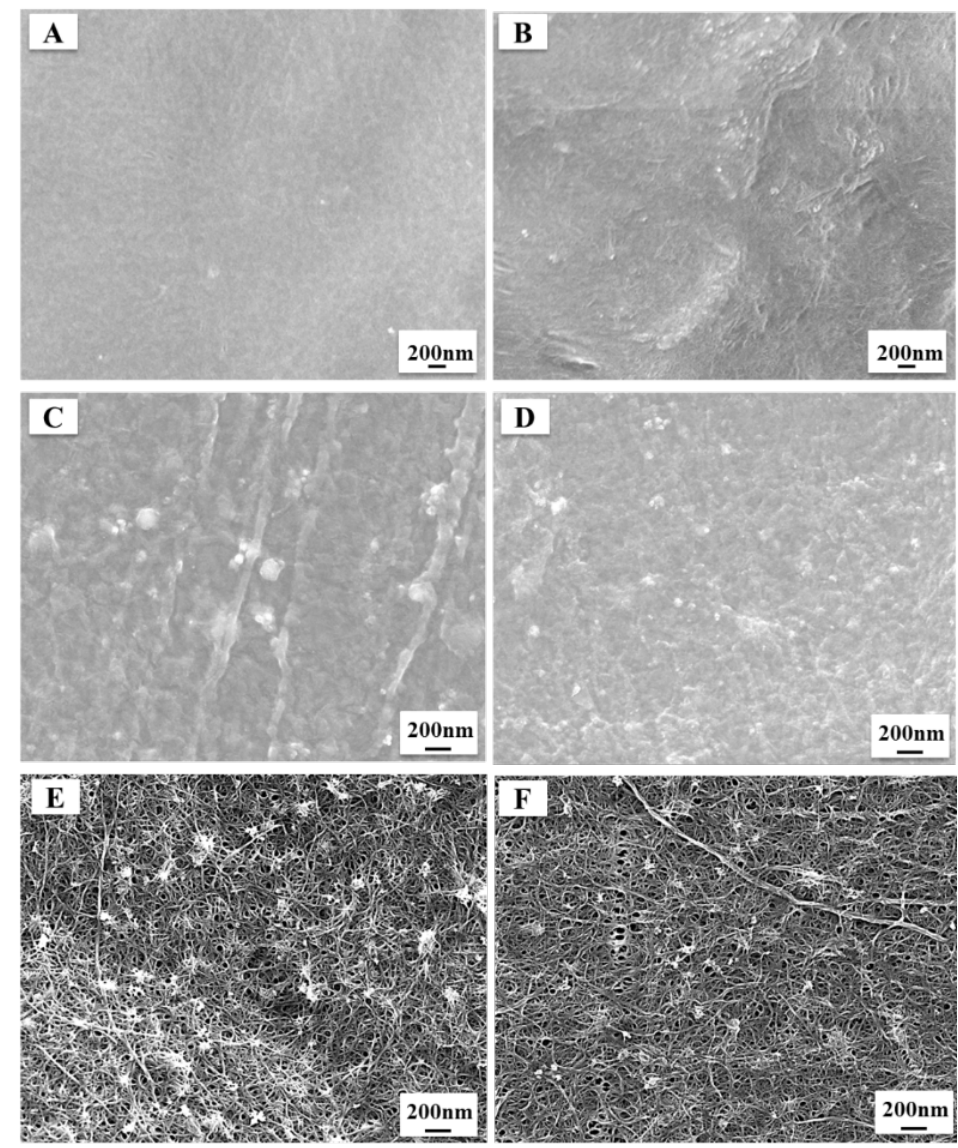

Figure 1. Fractured cross section (A) and top-view (C) SEM images of $10 \% \mathrm{ChNF} / \mathrm{PEO}$ composites. Fractured cross section (B) and top-view (D) SEM images of $20 \%$ ChNF/PEO composites. Top-view SEM images of dried solvent-washed 10\% (E) and $20 \%$ (F) ChNF/PEO composites.

\subsection{Mechanical Properties of ChNF/PEO Nano- composite Films}

Nanofibrous fillers can lead to composite materials with better mechanical properties than those of the neat polymers. However, their reinforcing effect depends on many factors, including filler morphologies, filler concentration and filler dispersion in matrices and filler adhesion with matrices.[1-7, 25-32] The mechanical properties of neat PEO and $\mathrm{ChNF} /$ PEO composite films are shown in Figure 2. Interestingly, the elastic modulus and tensile strength increased significantly with increasing ChNF loading, up to a factor of $\sim 3$ times for $20 \%$ ChNF compared to neat PEO. It is worth noting that a previous study by $\mathrm{Xu}$ et al. on the reinforcing effects of cellulose nanocrystals and cellulose nanofibrils in PEO matrices displayed a maximum at 7\% loading of the nanofibrous fillers.[32] The authors found that the tensile strength and modulus of the prepared composites initially increased when loading fillers up to $7 \%$ and then decreased as cellulose content further increased.
They further reasoned that the decreases in mechanical properties at higher filler loading resulted from filler agglomeration.[32] In contrast, the ChNFs obtained through a high-pressure homogenizer in this work exhibited a more uniform size, less entanglement and smaller bundles and were more well dispersed in the PEO matrix (Figure 1 and S1). Herein, the continuous increases in tensile strength and modulus of ChNF/PEO composites with up to $20 \% \mathrm{ChNF}$ loading are likely due to ChNFs' high mechanical properties, strong adhesion between PEO and ChNFs (as demonstrated below in FTIR and adhesion results), and good dispersion of ChNFs in the PEO matrix, resulting in efficient stress transfer within the ChNF network structure observed in SEM above. It is possible that beyond $20 \% \mathrm{ChNF}$ loading filler agglomeration might be observed, leading to a maximum in modulus and strength. However, due to difficulties in drying films at loadings beyond $20 \%$ ChNF using the solvent casting method (due to extra water added in the ChNF suspension), we did not examine loading above $20 \%$ ChNF.

On the other hand, Figure $2 \mathrm{~d}$ shows that strain-atbreak of the materials increased and then decreased as ChNF loading increased. Strain hardening was observed for composites comprising 5\%,10\% and 15\% ChNF, likely due to the alignment of the ChNFs during sample elongation. In contrast, the $20 \% \mathrm{ChNF}$ composite did not show strain hardening behavior.

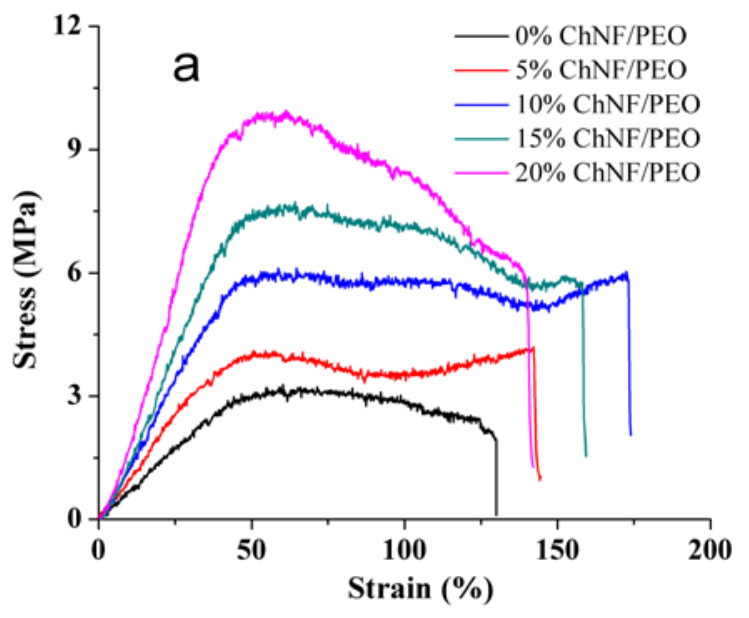



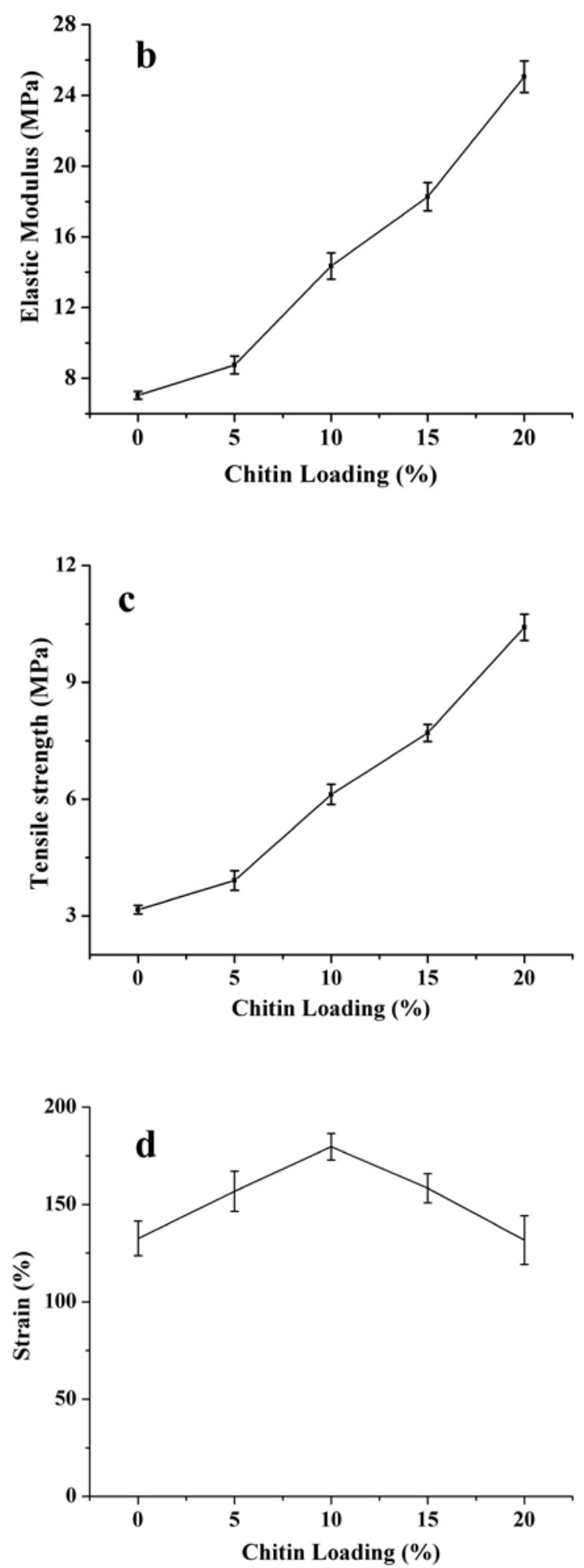

Figure 2. (a) Representative stress-strain curves, (b) elastic modulus, (c) tensile strength and (d) strain-at-break of neat PEO and $\mathrm{ChNF} / \mathrm{PEO}$ composites.

\subsection{FTIR of ChNF/PEO Nanocomposite Films}

In terms of chemical structures of chitin and $\mathrm{PEO}$, it is expected that hydrogen bonds may form between them since the ether oxygen $(\mathrm{C}-\mathrm{O}-\mathrm{C})$ in
$\mathrm{PEO}$ is a hydrogen bond acceptor and there are $-\mathrm{OH}$ and $-\mathrm{NH}$ hydrogen bond donors in chitin. Infrared spectroscopy is a highly effective method for investigating hydrogen bond interactions in blend composites.[33] As shown in Figure 3, the characteristic absorption bands for PEO are detected at 1095 and $2878 \mathrm{~cm}^{-1}$, which are attributed to $\mathrm{C}-\mathrm{O}-\mathrm{C}$ stretching and $\mathrm{CH}_{2}$ stretching, respectively.[33, 34] The characteristic peaks of chitin such as the amide band I at 1654 and $1620 \mathrm{~cm}^{-1}$ and the amide band II at 1554 $\mathrm{cm}^{-1}$ are observed.[8, 9] All these characteristic peaks from chitin and PEO can be detected in all the composites. With decreasing ChNF loading from 100\% to $5 \%$, the amide I and amide II shift to higher frequencies from 1620 to $1628 \mathrm{~cm}^{-1}$ and from 1554 to 1562 $\mathrm{cm}^{-1}$, respectively (Figure 4). This suggests that ChNF-ChNF hydrogen bonds involving the amide nitrogen have been disrupted after addition of PEO by formation of hydrogen bonds between surface$\mathrm{NH}$ groups on ChNF fibers and the ether oxygen of PEO. We note that the h-bonds between $\mathrm{O}-\mathrm{H}^{\cdots} \mathrm{N}(29$ $\mathrm{kJ} / \mathrm{mol}$ ) and $\mathrm{N}-\mathrm{H}^{\cdots} \mathrm{N}(13 \mathrm{~kJ} / \mathrm{mol})$ (representative of ChNF-ChNF) are stronger than $\mathrm{N}-\mathrm{H}^{\cdots} \mathrm{O}(8 \mathrm{~kJ} / \mathrm{mol})$ (representative of ChNF-PEO).[35, 36] Thus the shift to higher amide frequencies is consistent with the expectation of some fraction of weaker h-bonds between PEO to ChNF.

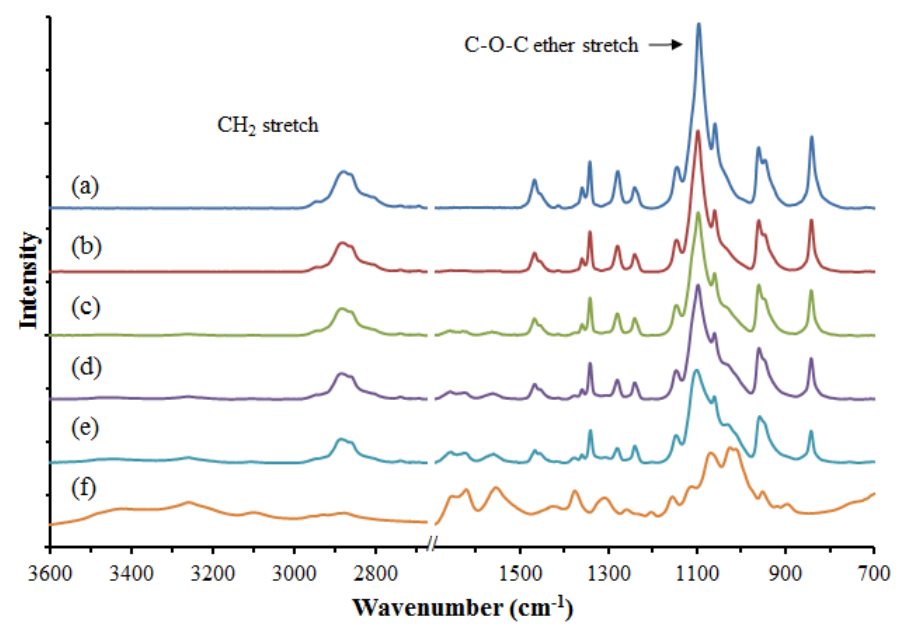

Figure 3. FTIR spectra of each composite films: (a) neat PEO, (b) $5 \% \mathrm{ChNF} / 95 \% \mathrm{PEO}$, (c) $10 \% \mathrm{ChNF} / 90 \% \mathrm{PEO}$, (d) $15 \%$ $\mathrm{ChNF} / 85 \% \mathrm{PEO}$, (e) $20 \% \mathrm{ChNF} / 80 \% \mathrm{PEO}$, and (f) neat ChNF. 


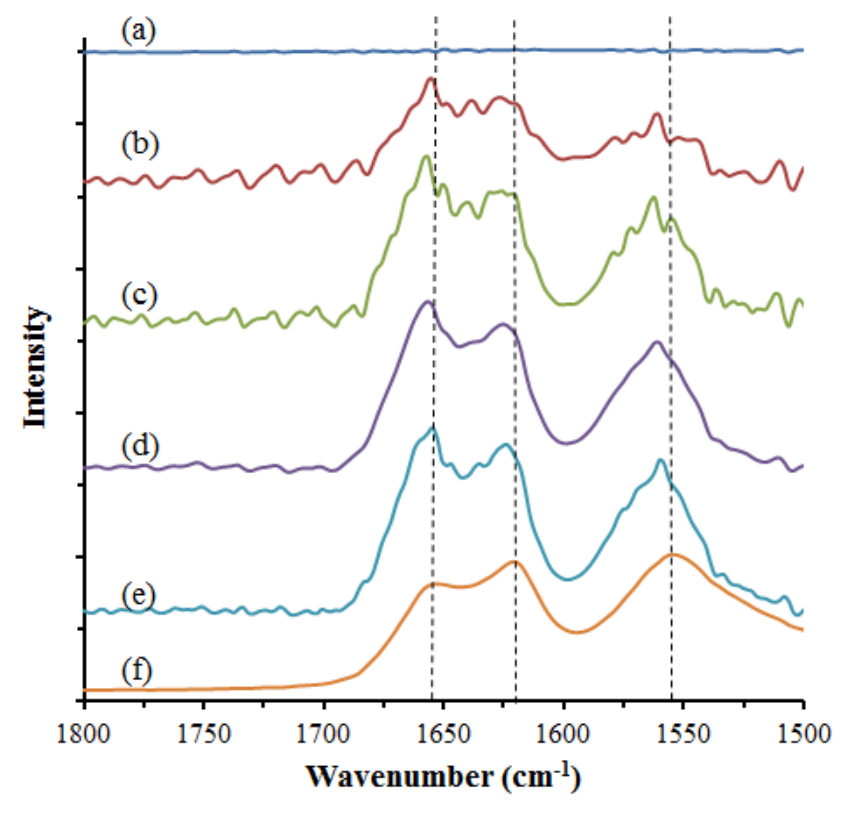

Figure 4. FTIR spectra of composite films in the 1500 to 1800 $\mathrm{cm}^{-1}$ region: (a) neat $\mathrm{PEO}$, (b) 5\% $\mathrm{ChNF} / 95 \% \mathrm{PEO}$, (c) $10 \%$ $\mathrm{ChNF} / 90 \% \quad \mathrm{PEO}, \quad$ (d) $15 \% \quad \mathrm{ChNF} / 85 \% \quad \mathrm{PEO}, \quad$ (e) $20 \%$ $\mathrm{ChNF} / 80 \%$ PEO, and (f) neat ChNF.

\subsection{Interaction of ChNF with Polymer Films}

An alternative possibility is that ChNF-ChNF hbonds are broken during fiber dispersion without any new PEO-ChNF h-bonds formed, which is difficult to ascertain using ATR-FTIR alone. To provide additional characterization of the nature of ChNF adhesion with PEO, adhesion force measurements were conducted using an AFM colloidal probe method. Figure 5 shows the morphologies of the ChNFcoated PS particle that was attached to the tipless cantilever. The adsorption of ChNFs on the PS surface was driven by their electrostatic attraction, where the PS particle has a negative charge due to surface sulfate groups and $\mathrm{ChNF}$ has positive charge because of protonated $-\mathrm{NH}_{3}{ }^{+}$groups. Five kinds of polymer substrates (PE, PS, PVAc, PVOH and PEO) were chosen to examine the effect of surface chemistry on the adhesion forces of ChNFs. The surface roughness, contact angles and calculated surface tension components of these polymers are listed in Tables 1-3. As shown in Table 3, PE and PS are essentially apolar, while PEO, PVAc and PVOH have large Lewis basic components, where lone electron pairs are provided by ether, carbonyl and hydroxyl groups, respectively. These surface tension results are consistent with polarity considerations of the molecular structures.
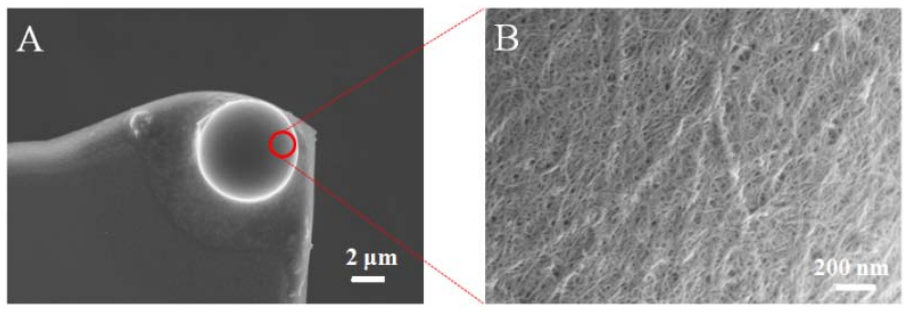

Figure 5. (A) SEM image of an AFM probe of ChNF-coated PS particle after all AFM force measurements; (B) Enlarged SEM image of surface morphology of ChNF-coated PS particle.

As shown in Figure 6, the adhesion forces for bare PS were independent of the polymer surface types. (Figure S3 shows the typical raw forcedistance curves for ChNF-coated PS particles on varied polymer surfaces, which indicates that their interactions are in a short-range $(<5 \mathrm{~nm})$.) This is consistent with the expectation that adhesion between PS and the polymer surfaces is governed by VDW forces. Furthermore, according to the Hamaker model, VDW adhesion between a particle and a flat surface depends on the Hamaker constant and a contact radius, which should be approximated well by the Hamaker model.[37]

$$
F_{\text {Hamaker }}=\frac{A R}{6 d^{2}}
$$

Since the values of the Hamaker constant for these five polymers are very close $\left(\sim 8-9 \times 10^{-20} \mathrm{~J}\right)$, the VDW forces are determined largely by the contact radius.[38] Therefore, the similar VDW adhesion forces between bare PS and these polymer surfaces suggest that the small variation in surface roughness of these polymers (Table 1) didn't affect their contact radii.

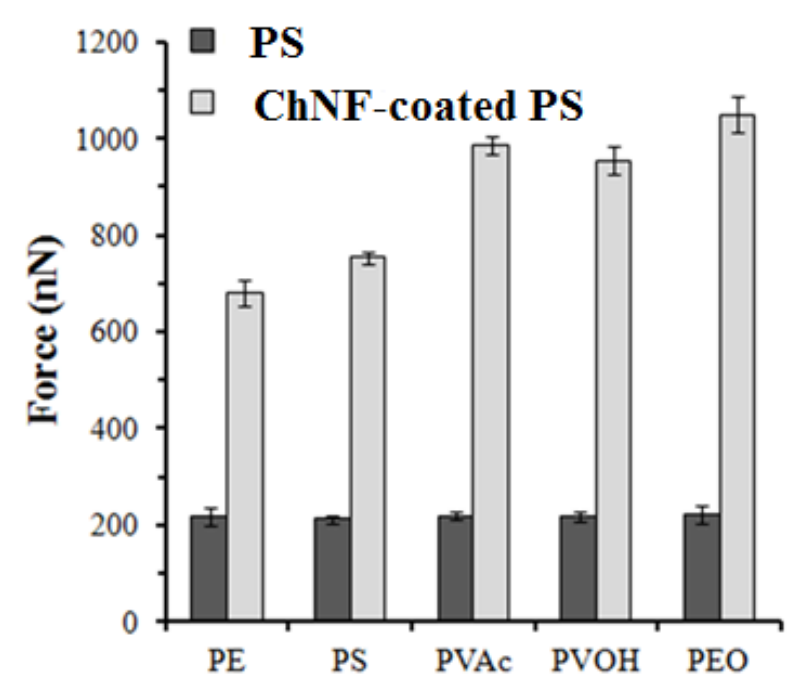

Figure 6. Adhesion forces for ChNF-coated and bare PS particles on various polymer surfaces. 
Table 1. Surface roughness of the various polymer surfaces.

\begin{tabular}{llllll}
\hline Surface & PE & PS & PVAc & PVOH & PEO \\
\hline $\mathrm{Ra}(\mathrm{nm})$ & $4.2 \pm 0.8$ & $2.4 \pm 0.4$ & $1.5 \pm 0.3$ & $1.6 \pm 0.4$ & $8.3 \pm 2.2$ \\
$\mathrm{rms}(\mathrm{nm})$ & $4.3 \pm 0.8$ & $2.8 \pm 0.4$ & $1.7 \pm 0.3$ & $1.9 \pm 0.4$ & $8.7 \pm 2.4$ \\
\hline
\end{tabular}

In contrast, the adhesion forces for ChNF-coated PS were all higher than PS alone, and they varied with different polymer surfaces (Figure 6). The marked increase in adhesion for ChNF-coated PS on $\mathrm{PE}$ and PS, apolar surfaces, is indicative of an increase in contact area for the ChNF-coated PS probes, which is consistent with the added roughness due to the ChNF coating observed in Figure 5. PE, the most apolar in the series, shows the lowest adhesion force value, while PVAc, PVOH, and PEO with high Lewis basic components possess higher adhesion forces. Typically, the short-range interaction $(<5$ $\mathrm{nm}$ ) includes dispersion (nonpolar, VDW force) and non-dispersion force (polar, acid-base interaction). Since the $\gamma^{\mathrm{vW}}$ of these polymer surfaces are not significatly different (Table 3), the VDW forces for $\mathrm{ChNF}$ are almost independent of polymer surface types. Therefore, the differences in adhesion forces suggest that Lewis acidic and basic components of the polymer surfaces play an important role in adhesion with ChNF.

Assuming van Oss and Good's Lifshitz-van der Waals acid-base theory for the solid (chitin surface, $\mathrm{Ch}$ )-solid (polymer surface, P) interface, the relationship of the adhesion force with the surface energy of the polymers can be expressed as:

$$
F_{a d} \propto a \sqrt{\gamma_{C h}^{v w} \gamma_{P}^{v w}}+b \sqrt{\gamma_{C h}^{+} \gamma_{P}^{-}}+c \sqrt{\gamma_{C h}^{-} \gamma_{P}^{+}}
$$

where, $F_{a d}$ is the experimentally determined adhesion force, and $a, b$ and $c$ are coefficients scaling the VDW and acidic-basic contributions, respectively. Since the VDW components of the five polymers $\left(\gamma_{P}{ }^{\mathrm{vW}}\right)$ are similar, equation 4 can be simplified to:

$$
F_{a d} \propto A+b \sqrt{\gamma_{C h}^{+} \gamma_{P}^{-}}+c \sqrt{\gamma_{C h}^{-} \gamma_{P}^{+}}
$$

where $A$ is a constant representing VDW-driven adhesion. We fitted the adhesion force data to equation 5, which is shown as a plane in Figure 7. Fitting all five polymers led to a correlation coefficient of $r^{2}$ $\sim 0.88$, suggesting that the differences of total adhesion forces on varied surfaces can be explained by acid-base (h-bonding interactions) of the polymer surfaces. Further, the relative contributions of acidic and basic components are not too different, $b / c=$ 1.46. Carbonyl, hydroxyl and ether oxygens in the polymers that possess them act as the major electron donor components and are able to form hydrogen bonds with $-\mathrm{OH},-\mathrm{NH}$ and $-\mathrm{NH}_{2}$ groups on the chitin structures. Since PEO has the largest $\gamma^{-}$value, indicative of its electron donors (hydrogen bond acceptors), and because chitin has protons that can serve as hydrogen bond donors, such as $-\mathrm{OH},-\mathrm{NH}-$ and $-\mathrm{NH}_{2}$, we conclude that the acid-base interaction between PEO and chitin are hydrogen bonds. This is consistent with FTIR data presented above.

Table 2. Contact angles $\left({ }^{\circ}\right)$ of polymer surfaces with three testing liquids.

\begin{tabular}{llll}
\hline Substrate & Water & glycerol & diiodomethane \\
\hline PE & $105.2 \pm 4.0$ & $87.3 \pm 2.1$ & $52.8 \pm 2.0$ \\
PS & $101.1 \pm 3.0$ & $84.2 \pm 2.0$ & $33.6 \pm 2.0$ \\
PVAc & $60.7 \pm 2.9$ & $70.7 \pm 3.0$ & $37.8 \pm 3.0$ \\
PVOH & $46.2 \pm 1.4$ & $44.0 \pm 1.3$ & $40.4 \pm 1.4$ \\
PEO & $19.0 \pm 4.0$ & $46.8 \pm 2.0$ & $33.8 \pm 2.5$ \\
Chitin & $56.7 \pm 1.4$ & $46.7 \pm 1.4$ & $37.4 \pm 2.0$ \\
\hline
\end{tabular}

Table 3. Surface tension components $\left(\mathrm{mJ} / \mathrm{m}^{2}\right)$ of various polymer surfaces.

\begin{tabular}{lllll}
\hline Surface & $\gamma^{\mathrm{vW}}$ & $\gamma^{+}$ & $\gamma^{-}$ & $\gamma$ \\
\hline PE & 33 & 0 & 0 & 33.0 \\
PS & 43 & 0.2 & 0.4 & 43.2 \\
PVAc & 41 & 0.6 & 16.7 & 46.8 \\
PVOH & 39 & 1.1 & 28.8 & 50.4 \\
PEO & 43 & 0 & 64.0 & 43.0 \\
Chitin & 41 & 1.3 & 17.1 & 50.4 \\
\hline
\end{tabular}




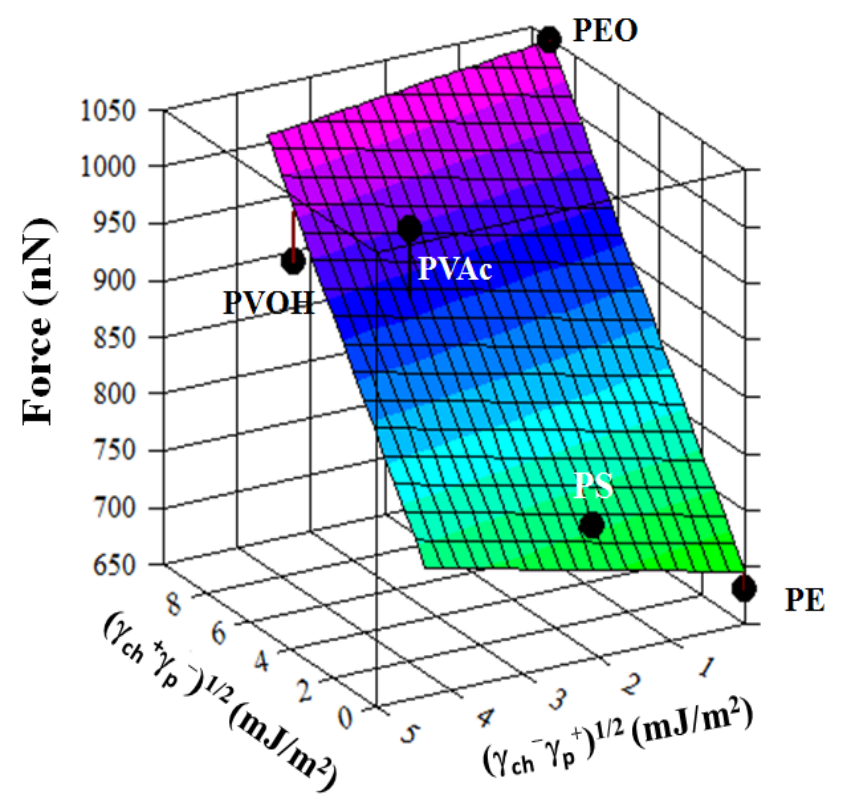

Figure 7. Fitting of the planar model of equation 4 to adhesion force data for ChNF-coated PS colloidal probes with five substrates of varying polarity.

\subsection{DSC Analysis}

The melting temperature $\left(T_{m}\right)$, enthalpy of fusion and crystallinity $\left(X_{c}\right)$ of neat $\mathrm{PEO}$ and $\mathrm{ChNF} / \mathrm{PEO}$ composite films were measured using DSC. The degree of crystallinity of these samples was calculated according to equation $6:[39]$

$$
X_{c}=\frac{\Delta H_{i}}{f_{i} \Delta H_{i}^{m}} \times 100 \%
$$

where, $\Delta H_{i}$ is the enthalpy of fusion of the prepared PEO samples, obtained from DSC measurements, $f_{i}$ is the mass fraction of $\mathrm{PEO}$ in the composite, and $\Delta H_{i}^{m}$ is the enthalpy of fusion of $100 \%$ crystalline PEO, which is $205 \mathrm{~J} / \mathrm{g}$.[39] The neat PEO has a melting temperature of $67.3{ }^{\circ} \mathrm{C}$ and crystallinity of $86.3 \%$ (Table 4), and the crystallinity and melting temperature of PEO decreased with increasing ChNF loading. We reason that the dispersion of $\mathrm{ChNF}$ in the PEO matrix and strong interactions between ChNF and $\mathrm{PEO}$ hindered chain diffusion and folding during PEO crystallization, resulting in the low crystallinity for composite samples. The neat PEO has a $\mathrm{T}_{\mathrm{g}}$ of $56.2{ }^{\circ} \mathrm{C}$ while the $\mathrm{T} g$ of $5 \%, 10 \%$ and $15 \%$ ChNF/PEO composites are $-49.2,-48.7$ and $-48.4{ }^{\circ} \mathrm{C}$, respectively. The increase in glass transition temperature and decrease in crystallinity with ChNF content for the composites is consistent with the strong adhesion between $\mathrm{ChNF}$ and $\mathrm{PEO}$ that likely restricts PEO chain mobility.

Table 3. Melting temperature and crystallinities of neat $\mathrm{PEO}$ and $\mathrm{PEO} / \mathrm{ChNF}$ composite.

\begin{tabular}{llll}
\hline Sample & $\mathrm{T}_{\mathrm{m}}\left({ }^{\circ} \mathrm{C}\right)$ & $\Delta \mathrm{H}(\mathrm{J} / \mathrm{g})$ & $\mathrm{X}_{\mathrm{c}}(\%)$ \\
\hline Neat PEO & 67.3 & 177.0 & 86.3 \\
$5 \%$ Chitin/PEO & 65.8 & 165.5 & 85.0 \\
$10 \%$ Chitin/PEO & 64.0 & 152.3 & 82.6 \\
$15 \%$ Chitin/PEO & 62.7 & 137.4 & 78.9 \\
20\% Chitin/PEO & 62.0 & 122.3 & 74.6 \\
\hline
\end{tabular}

\section{Conclusions}

In this study, ChNF/PEO nanocomposites were successfully fabricated by an aqueous solution casting method and the structure-property relationships of the nanocomposites were investigated. ChNFs were dispersed well and formed interconnected network structures in the PEO matrix. Compared to neat $\mathrm{PEO}$, the tensile strength and elastic modulus of the nanocomposites increased $\sim 3$ times at $20 \% \mathrm{ChNF}$ loading. In addition to ChNFs' high mechanical properties, the strong interactions between $\mathrm{ChNF}$ and PEO, and the ChNF network structure played important roles in efficient stress transfer from matrix to fiber and from fiber to fiber. The ATR-FTIR and AFM colloidal probe adhesion measurements support the conclusion that $\mathrm{ChNF}$ has strong h-bond and VDW-driven adhesion with itself and with PEO

The crystallinities of PEO in composites are lower than that of neat PEO, which may be attributed to dispersion of $\mathrm{ChNF}$ in the PEO matrix and strong interactions between $\mathrm{ChNF}$ and $\mathrm{PEO}$ that restrict the chain mobility during PEO crystallization. Processing ChNFs in suspensions with slight acidity and water-soluble polymers appears to support the formation of highly interconnected networks that makes ChNF-polymer composites excellent candidates for reinforced, light-weight, renewable materials.

\section{Author Contributions}

The manuscript was written through contributions of all authors. All authors have given approval to the final version of the manuscript.

$\S$ These authors contributed equally.

\section{Acknowledgments}

We are thankful to the Renewable Bioproducts Institute (Georgia Institute of Technology) and the Air Force Office of Scientific Research (Grant \# 
FA9550-10-1-0555) for financial support of this research.

\section{References}

[1] T. Chatterjee, K. Yurekli, V.G. Hadjiev and R. Krishnamoorti, Single-walled carbon nanotube dispersions in poly(ethylene oxide), Advanced Functional Materials, 15 (2005) 1832-1838.

[2] L.A. Goettler, K.Y. Lee and H. Thakkar, Layered silicate reinforced polymer nanocomposites: Development and applications, Polymer Reviews, 47 (2007) 291-317.

[3] T. Chatterjee, C.A. Mitchell, V.G. Hadjiev and R. Krishnamoorti, Hierarchical polymernanotube composites, Advanced Materials, 19 (2007) 3850-+.

[4] S.Y. Fu, X.Q. Feng, B. Lauke and Y.W. Mai, Effects of particle size, particle/matrix interface adhesion and particle loading on mechanical properties of particulate-polymer composites, Composites Part B-Engineering, 39 (2008) 933961.

[5] B. Chen, J.R.G. Evans, H.C. Greenwell, P. Boulet, P.V. Coveney, A.A. Bowden and A. Whiting, A critical appraisal of polymer-clay nanocomposites, Chemical Society Reviews, 37 (2008) 568-594.

[6] S. Bredeau, S. Peeterbroeck, D. Bonduel, M. Alexandre and P. Dubois, From carbon nanotube coatings to high-performance polymer nanocomposites, Polymer International, 57 (2008) 547-553.

[7] D. Klemm, F. Kramer, S. Moritz, T. Lindstrom, M. Ankerfors, D. Gray and A. Dorris, Nanocelluloses: A New Family of Nature-Based Materials, Angewandte Chemie-International Edition, 50 (2011) 5438-5466.

[8] M. Rinaudo, Chitin and chitosan: Properties and applications, Progress in Polymer Science, 31 (2006) 603-632.

[9] C.K.S. Pillai, W. Paul and C.P. Sharma, Chitin and chitosan polymers: Chemistry, solubility and fiber formation, Progress in Polymer Science, 34 (2009) 641-678.

[10] J.-B. Zeng, Y.-S. He, S.-L. Li and Y.-Z. Wang, Chitin Whiskers: An Overview, Biomacromolecules, 13 (2012) 1-11.

[11] J. Wu, K. Zhang, N. Girouard and J.C. Meredith, Facile Route to Produce Chitin
Nanofibers as Precursors for Flexible and Transparent Gas Barrier Materials, Biomacromolecules, 15 (2014) 4614-4620.

[12] J. Wu and J.C. Meredith, Assembly of Chitin Nanofibers into Porous Biomimetic Structures via Freeze Drying, Acs Macro Letters, 3 (2014) 185-190.

[13] M. Trollsås and J.L. Hedrick, Dendrimer-like Star Polymers, Journal of the American Chemical Society, 120 (1998) 4644-4651.

[14] A.C. Albertsson and I.K. Varma, Recent developments in ring opening polymerization of lactones for biomedical applications, Biomacromolecules, 4 (2003) 1466-1486.

[15] K. Gopalan Nair and A. Dufresne, Crab shell chitin whisker reinforced natural rubber nanocomposites. 1. Processing and swelling behavior, Biomacromolecules, 4 (2003) 657665.

[16] S. Ifuku, M. Nogi, K. Abe, M. Yoshioka, M. Morimoto, H. Saimoto and H. Yano, Preparation of chitin nanofibers with a uniform width as alpha-chitin from crab shells, Biomacromolecules, 10 (2009) 1584-1588.

[17] J.C. Meredith, A. Karim and E.J. Amis, Highthroughput measurement of polymer blend phase behavior, Macromolecules, 33 (2000) 57605762 .

[18] B.J.R. Thio, J.H. Lee and J.C. Meredith, Characterization of Ragweed Pollen Adhesion to Polyamides and Polystyrene Using Atomic Force Microscopy, Environmental Science \& Technology, 43 (2009) 4308-4313.

[19] J.L. Hutter and J. Bechhoefer, Calibration of Atomic-Force Microscope Tips, Rev. Sci. Instrum., 64 (1993) 1868-1873.

[20] R.J. Good and C.J. van Oss, Modern Approach of Wettability: Theory and Applications, Plenum Press, New York, 1991.

[21] H.J. Busscher and J. Arends, Determination of the Surface Forces Gamma-S-D and Gamma-S-P from Contact-Angle Measurements on Polymers and Dental Enamel, Journal of Colloid and Interface Science, 81 (1981) 75-79.

[22] K.X. Ma, T.S. Chung and R.J. Good, Surface energy of thermotropic liquid crystalline polyesters and polyesteramide, Journal of Polymer Science Part B-Polymer Physics, 36 (1998).

[23] J.L. Sormana and J.C. Meredith, Highthroughput discovery of structure-mechanical 
property relationships for segmented poly(urethane-urea)s, Macromolecules, 37 (2004) 2186-2195.

[24] J.L. Sormana, S. Chattopadhyay and J.C. Meredith, High-throughput mechanical characterization of free-standing polymer films, Review of Scientific Instruments, 76 (2005).

[25] M. Samir, F. Alloin, M. Paillet and A. Dufresne, Tangling effect in fibrillated cellulose reinforced nanocomposites, Macromolecules, 37 (2004) 4313-4316.

[26] M.O. Seydibeyoglu and K. Oksman, Novel nanocomposites based on polyurethane and micro fibrillated cellulose, Composites Science and Technology, 68 (2008) 908-914.

[27] A. Iwatake, M. Nogi and H. Yano, Cellulose nanofiber-reinforced polylactic acid, Composites Science and Technology, 68 (2008) 2103-2106.

[28] F. Dalmas, J.-Y. Cavaille, C. Gauthier, L. Chazeau and R. Dendievel, Viscoelastic behavior and electrical properties of flexible nanofiber filled polymer nanocomposites. Influence of processing conditions, Composites Science and Technology, 67 (2007) 829-839.

[29] Q. Cheng, S. Wang and T.G. Rials, Poly(vinyl alcohol) nanocomposites reinforced with cellulose fibrils isolated by high intensity ultrasonication, Composites Part a-Applied Science and Manufacturing, 40 (2009) 218-224.

[30] G. Chinga-Carrasco, Cellulose fibres, nanofibrils and microfibrils: The morphological sequence of MFC components from a plant physiology and fibre technology point of view, Nanoscale Research Letters, 6 (2011).

[31] K.-Y. Lee, T. Tammelin, K. Schulfter, H. Kiiskinen, J. Samela and A. Bismarck, High Performance Cellulose Nanocomposites: Comparing the Reinforcing Ability of Bacterial Cellulose and Nanofibrillated Cellulose, Acs
Applied Materials \& Interfaces, 4 (2012) 40784086.

[32] X. Xu, F. Liu, L. Jiang, J.Y. Zhu, D. Haagenson and D.P. Wiesenborn, Cellulose Nanocrystals vs. Cellulose Nanofibrils: A Comparative Study on Their Microstructures and Effects as Polymer Reinforcing Agents, Acs Applied Materials \& Interfaces, 5 (2013) 2999-3009.

[33] J. Jin, P. Hassanzadeh, G. Perotto, W. Sun, M.A. Brenckle, D. Kaplan, F.G. Omenetto and M. Rolandi, A Biomimetic Composite from Solution Self-Assembly of Chitin Nanofibers in a Silk Fibroin Matrix, Advanced Materials, 25 (2013) 4482-4487.

[34] E.E. Brown and M.-P.G. Laborie, Bloengineering bacterial cellulose/poly(ethylene oxide) nanocomposites, Biomacromolecules, 8 (2007) 3074-3081.

[35] J. Emsley, Very Strong Hydrogen-Bonding, Chemical Society Reviews, 9 (1980) 91-124.

[36] J.W. Larson and T.B. McMahon, Gas-phase bihalide and pseudobihalide ions. An ion cyclotron resonance determination of hydrogen bond energies in $\mathrm{XHY}$ - species $(\mathrm{X}, \mathrm{Y}=\mathrm{F}, \mathrm{Cl}$, $\mathrm{Br}, \mathrm{CN})$, Inorganic Chemistry, 23 (1984) 20292033.

[37] J. Israelachvili, Intermolecular and Surface Forces, Acadamic Press, London, 1992.

[38] H. Lin, I. Gomez and J.C. Meredith, Pollenkitt Wetting Mechanism Enables Species-Specific Tunable Pollen Adhesion, Langmuir, 29 (2013) 3012-3023.

[39] I.T. Kim, J.H. Lee, M.L. Shofner, K. Jacob and R. Tannenbaum, Crystallization kinetics and anisotropic properties of polyethylene oxide/magnetic carbon nanotubes composite films, Polymer, 53 (2012) 2402-2411. 
